DOI: 10.1002/adsc.201((will be filled in by the editorial staff))

\title{
Brønsted Acid-Catalyzed Straightforward Synthesis of Benzo[b]carbazoles from 2,3-Unsubstituted Indoles
}

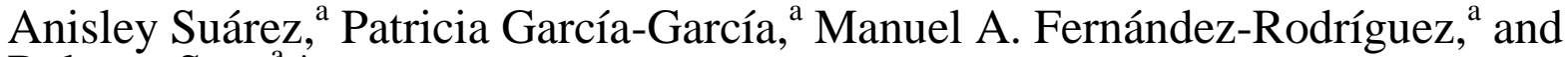 \\ Roberto Sanz $z^{\mathrm{a}, *}$
}

a Área de Química Orgánica, Departamento de Química, Facultad de Ciencias, Universidad de Burgos, Pza. Misael

Bañuelos s/n, 09001-Burgos (Spain)

Fax: (+34)-947-258831; E-mail: rsd@ubu.es

Received: ((will be filled in by the editorial staff))

Supporting information for this article is available on the WWW under http://dx.doi.org/10.1002/adsc.201\#\#\#\#\#\#.

\begin{abstract}
Described is a general and efficient synthesis of valuable benzo $[b]$ carbazoles by Brønsted acid-catalyzed reaction between simple $\mathrm{C} 2, \mathrm{C} 3$-unsubstituted indoles and $o$-( $\alpha$-(hydroxy)benzyl)benzaldehyde acetals. Highly selective migration processes are involved as key steps in the overall cascade sequence that implies the one-pot formation of two new bonds and a cycle in a regioselective fashion.
\end{abstract}

Keywords: Brønsted acid catalysis; heterocycles; benzo[b]carbazoles; 1,2-alkyl shift; synthetic methods

Aryl- and heteroaryl-condensed carbazoles have attracted considerable interest because of their broad spectrum of useful biological activities. ${ }^{[1]}$ Among the benzocarbazole frameworks, annulated $[b]$ carbazoles, such as ellipticine and its derivatives, ${ }^{[2]}$ are relevant due to their interesting pharmacological activities ${ }^{[3]}$ as well as their utility in the field of material chemistry. ${ }^{[4]}$ Different approaches for accessing this type of heterocyclic compounds have been described, although most of them imply multi-step sequences and restricted substitution patterns. In this regard, benzo $[b]$ carbazoles are commonly constructed from either properly functionalized indoles or carbazoles, ${ }^{[5]}$ substituted naphthalenes, ${ }^{[6]}$ or more particularly from ketenimines and imidoyl selanides by radical cyclizations, ${ }^{[7]}$ or from ynamides by dehydro Diels-Alder reactions. ${ }^{[8]}$ However, to the best of our knowledge, there are not general methodologies developed for the direct synthesis of (hetero)aryl $[b]$ carbazoles starting from simple C2,C3unsubstituted indoles (Figure 1). ${ }^{[9]}$

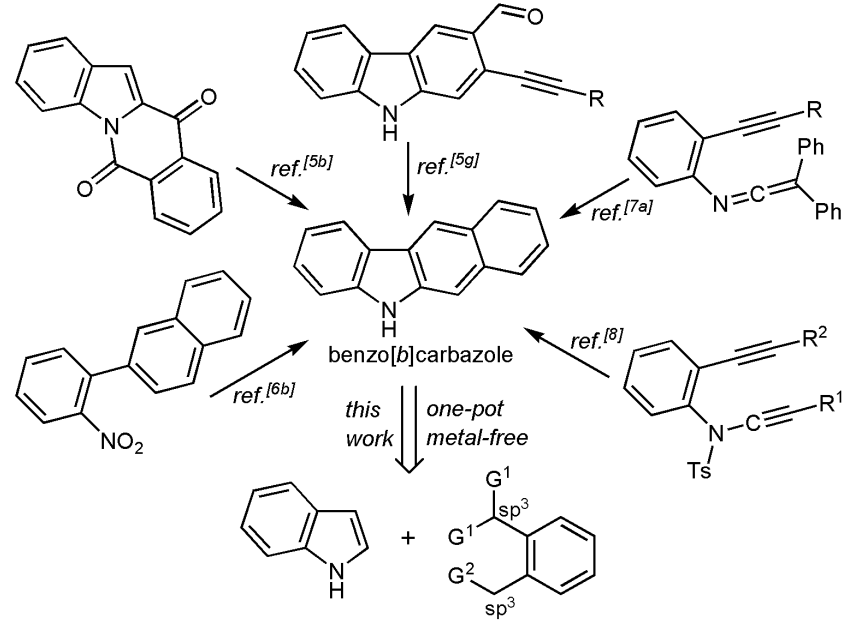

Figure 1. Synthetic routes to benzo[b]carbazoles.

On the other hand, the reaction of indoles with carbonyl derivatives, including acetals, ${ }^{[10]}$ in the presence of Lewis or protic acids is the most general method for the synthesis of symmetric 3,3'bisindolylmethanes (3,3'-BIMs) (II). Their formation is proposed to proceed through the intermediacy of an azafulvenium species such as $\mathbf{I}$ (with $\mathrm{Nu}=\mathrm{H}$ ) that undergoes further addition of a second indole molecule (Scheme 1). ${ }^{[1]}$ When intermediates I are functionalized with an additional nucleophilic group such as an electron-rich aromatic $(\mathrm{Nu}=\mathrm{Ar})$, a second Friedel-Crafts alkylation process can take place affording products like III (Scheme 1). ${ }^{[12]}$ Alternatively, it's well known that neutral alkylideneindolenine intermediates related with $\mathbf{I}(\mathrm{Nu}$ $=\mathrm{H}$ ), commonly formed from precursors having a suitable leaving group at the benzylic position of 3substituted indoles, are able to add external nucleophiles in a conjugate fashion. ${ }^{[13]}$

Herein we wish to report our results on the Brønsted acid-catalyzed reaction between indoles and aromatic aldehyde acetals possessing an $\alpha$ (hydroxy)benzyl group at the ortho-position, which enables a facile entry into the synthesis of 
regioselectively functionalized benzo[b]carbazoles through an unprecedented cascade sequence.

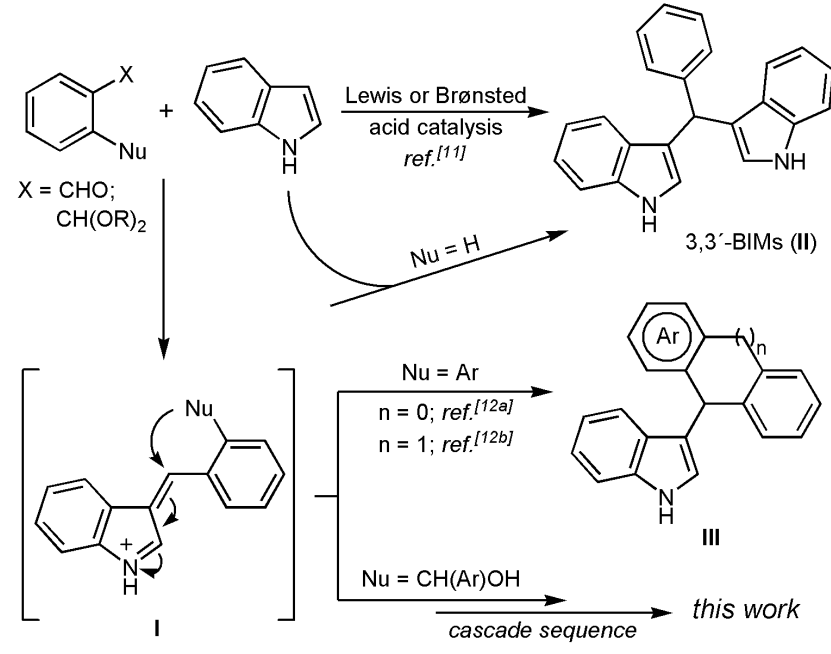

Scheme 1. Reported reactions of indoles and benzaldehyde derivatives: synthesis of 3,3'-BIMs (II) and C3carbocyclic-functionalized indoles (III).

In the last years, we have been interested in the development of new methodologies for the direct C3alkylation of indoles with alcohols. ${ }^{[14]}$ As part of this research, we study the reaction between $N$ methylindole 1a and (2(diethoxymethyl)phenyl)(phenyl)methanol $\quad \mathbf{2 a}^{[15]}$ using various acid catalysts (Scheme 2). The use of selected $\sigma$-Lewis acids, previously employed for the reaction of indoles with carbonyl derivatives, afforded isobenzofuran derivative 3aa, obtained as a mixture of diastereoisomers, as the only product without formation of the corresponding 3,3'-BIM. Surprisingly, the same reaction under Brønsted acid catalysis (20 mol\% of PTSA) selectively afforded a new product, the benzo[b]carbazole derivative 4aa, which was isolated in $78 \%$ yield. ${ }^{[16]}$ Remarkably, its formation formally involves two consecutive and selective Friedel-Crafts-type reactions of a C2,C3unsubstituted indole with a bis(electrophile) bearing an alcohol as $\mathrm{sp}^{3}$-type acceptor and an acetal as carbonyl-type acceptor. Interestingly, whereas the preparation of annulated indolic frameworks by the formal C2-hydroarylation of indoles with $\mathrm{sp}^{2}$ - or sptype acceptors, such as Michael substrates ${ }^{[17]}$ or goldactivated alkynes, ${ }^{[18]}$ has been well established, the corresponding reactions with $\mathrm{sp}^{3}$-type acceptors like halides or alcohols through the Ciamician-Plancher rearrangement have been scarcely reported. ${ }^{[19]}$

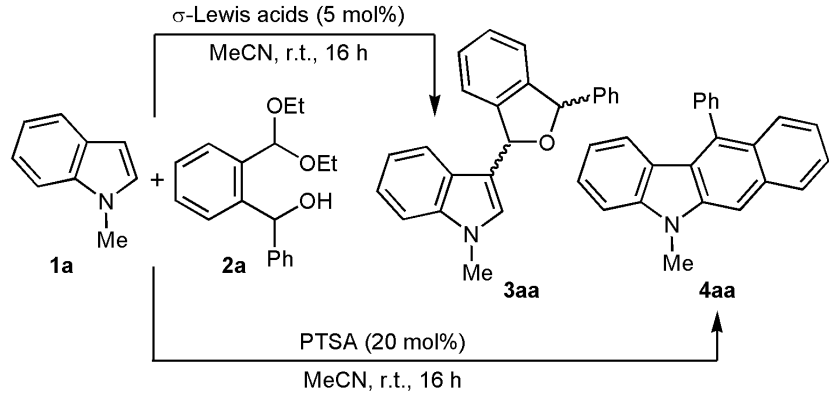

Scheme 2. Acid-catalyzed reaction of $N$-methylindole 1a with diethylacetal 2a. Selective formation of 3aa and 4aa.

Having found mild conditions to efficiently and directly access 4aa from readily available $N$ methylindole we decided to check if this methodology could be general for the synthesis of a variety of benzo $[b]$ carbazoles. First, $N$-methylindole 1a was treated with selected benzylic alcohols 2a-i, prepared from 2-lithiobenzaldehyde diethyl acetal and selected (hetero)aryl carboxaldehydes. As proved in Table 1, both EDG and EWG substituents on the Ar group, as well as heteroaromatics, were adequately tolerated in the process and 11 -aryl- $5 \mathrm{H}$ benzo $[b]$ carbazoles $\mathbf{4 a a - a i ^ { [ 2 0 ] }}$ were obtained typically in high yields. Reactions with highly activated benzylic alcohols bearing EDG groups proceed faster and with a lower amount of the Brønsted acid catalyst (entries 5-9 vs 1-4). Interestingly, no influence in the process or yield was observed by varying the acetal moiety as we determined in the synthesis of benzo $[b]$ carbazole 4ae from 2e and 2'e (entry 5). In addition, NH-indole $\mathbf{1 b}$ also reacted with selected hydroxyacetals $\mathbf{2}$ to furnish benzocarbazole derivatives 4ba-bi in good yields (entries 10-14). Moreover, even less nucleophilic 5-functionalized indoles 1c-f are able to participate in this reaction allowing the regioselective preparation of benzo $[b]$ carbazoles $\mathbf{4 c e}$ and $\mathbf{4 d f - f f}$ in moderate to high yields (entries 15-18). However, the presence of an aromatic group (Ar) as substituent in the starting alcohol 2 seems to be mandatory for the success of the reaction as substrates bearing alkyl $(n-\mathrm{Bu})$, cyclopropyl, $\quad(E)-\beta$-styrenyl $\quad(\mathrm{CH}=\mathrm{CHPh}), \quad$ or phenylethynyl $(\mathrm{C} \equiv \mathrm{CPh})$ gave only rise to decomposition products under the standard reaction conditions.

Table 1. Synthesis of benzo[b]carbazoles 4 .

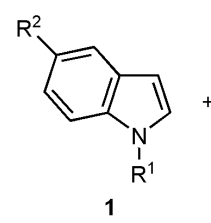<smiles>CCOC([Te])c1ccccc1C(OCC)OCC</smiles>

PTSA (20 mol\%)

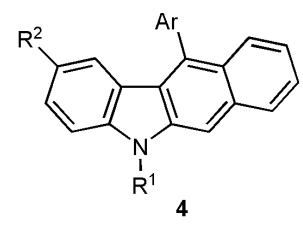




\begin{tabular}{|c|c|c|c|c|c|c|c|c|}
\hline Entry & 1 & $\mathrm{R}^{1}$ & $\mathrm{R}^{2}$ & 2 & $\mathrm{Ar}$ & $t[\mathrm{~h}]$ & Product & Yield $[\%]^{[\mathrm{a}]}$ \\
\hline 1 & 1a & $\mathrm{Me}$ & $\mathrm{H}$ & $2 \mathbf{a}$ & $\mathrm{Ph}$ & 16 & 4aa & 78 \\
\hline $2^{[b]}$ & $1 \mathbf{a}$ & $\mathrm{Me}$ & $\mathrm{H}$ & $2 \mathbf{b}$ & 4- $\mathrm{ClC}_{6} \mathrm{H}_{4}$ & 24 & 4ab & 89 \\
\hline $3^{[b]}$ & 1a & $\mathrm{Me}$ & $\mathrm{H}$ & $2 c$ & $4-\mathrm{BrC}_{6} \mathrm{H}_{4}$ & 16 & $4 a c$ & 65 \\
\hline $4^{[b]}$ & $1 a$ & $\mathrm{Me}$ & $\mathrm{H}$ & 2d & 2-Naphthyl & 3.5 & 4ad & 58 \\
\hline 5 & 1a & $\mathrm{Me}$ & $\mathrm{H}$ & $2 e$ & $4-\mathrm{MeOC}_{6} \mathrm{H}_{4}$ & 16 & $4 a e$ & $85^{[\mathrm{c}]}$ \\
\hline 6 & 1a & $\mathrm{Me}$ & $\mathrm{H}$ & $2 f$ & $2,3,4-(\mathrm{MeO})_{3} \mathrm{C}_{6} \mathrm{H}_{2}$ & 0.5 & 4af & 90 \\
\hline 7 & 1a & $\mathrm{Me}$ & $\mathrm{H}$ & $2 \mathrm{~g}$ & 2-Thienyl & 2 & $4 a g$ & 73 \\
\hline 8 & 1a & $\mathrm{Me}$ & $\mathrm{H}$ & $2 \mathrm{~h}$ & 3-Methyl-2-thienyl & 1.5 & 4ah & 71 \\
\hline 9 & 1a & $\mathrm{Me}$ & $\mathrm{H}$ & $2 \mathbf{i}$ & 5-Methyl-2-furyl & 2 & 4ai & $51^{[\mathrm{d}]}$ \\
\hline $10^{[\mathrm{b}]}$ & 1b & $\mathrm{H}$ & $\mathrm{H}$ & $2 a$ & $\mathrm{Ph}$ & 5 & $4 \mathrm{ba}$ & 65 \\
\hline $11^{[\mathrm{b}]}$ & $1 \mathrm{~b}$ & $\mathrm{H}$ & $\mathrm{H}$ & $2 d$ & 2-Naphthyl & 4 & $4 b d$ & 50 \\
\hline 12 & $1 \mathrm{~b}$ & $\mathrm{H}$ & $\mathrm{H}$ & $2 \mathrm{e}$ & $4-\mathrm{MeOC}_{6} \mathrm{H}_{4}$ & 16 & 4be & 92 \\
\hline 13 & 1b & $\mathrm{H}$ & $\mathrm{H}$ & $2 f$ & $2,3,4-(\mathrm{MeO})_{3} \mathrm{C}_{6} \mathrm{H}_{2}$ & 0.5 & $4 \mathrm{bf}$ & 98 \\
\hline 14 & 1b & $\mathrm{H}$ & $\mathrm{H}$ & $2 \mathbf{i}$ & 5-Methyl-2-furyl & 3.5 & $4 b i$ & $68^{[\mathrm{d}]}$ \\
\hline 15 & 1c & $\mathrm{H}$ & $\mathrm{Br}$ & $2 e$ & $4-\mathrm{MeOC}_{6} \mathrm{H}_{4}$ & 4 & 4ce & 48 \\
\hline 16 & 1d & $\mathrm{H}$ & $\mathrm{NO}_{2}$ & $2 f$ & $2,3,4-(\mathrm{MeO})_{3} \mathrm{C}_{6} \mathrm{H}_{2}$ & 1 & $4 d f$ & 85 \\
\hline 17 & $1 \mathrm{e}$ & $\mathrm{H}$ & $\mathrm{Cl}$ & $2 f$ & $2,3,4-(\mathrm{MeO})_{3} \mathrm{C}_{6} \mathrm{H}_{2}$ & 1 & $4 \mathrm{ef}$ & 94 \\
\hline 18 & 1f & $\mathrm{H}$ & $\mathrm{CO}_{2} \mathrm{Me}$ & $2 f$ & $2,3,4-(\mathrm{MeO})_{3} \mathrm{C}_{6} \mathrm{H}_{2}$ & 1 & $\mathbf{4 f f}$ & 80 \\
\hline
\end{tabular}

${ }^{\text {[a] }}$ Yield of isolated products 4 based on the starting indole $1 .{ }^{[b]}$ Carried out with 50 mol\% of PTSA. ${ }^{[c]}$ Same yield was obtained in a related reaction starting from alcohol 2'e bearing an ethylene acetal moiety instead of a diethyl acetal one. ${ }^{\text {[d] }}$ $7-10 \%$ of the corresponding 6-arylbenzo $[b]$ carbazole 5 was also isolated and characterized.

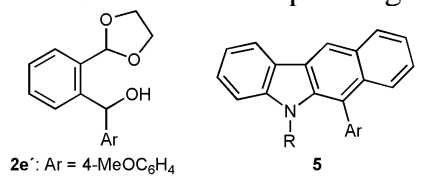

Remarkably, all these reactions selectively occurred to form 11-aryl-5H-benzo[b]carbazoles 4 while the corresponding regioisomeric 6-arylsubstituted benzo $[b]$ carbazoles $\mathbf{5}$ were only observed in trace to minor amounts in some cases (entries 9 and 14). Intrigued by these particular results, we decided to further explore this process by using hydroxyacetals $\mathbf{2 j}$-n functionalized with a methoxy group at the aryl ring that contains the acetal group and with different aryl groups at the benzylic position. Their reactions with $N$-methylindole 1a under the standard conditions, PTSA (20 mol\%) in $\mathrm{MeCN}$ at $\mathrm{RT}$, mainly gave rise to the expected 11-aryl-5Hbenzo[b]carbazoles 4 that could be isolated in synthetically useful yields (Table 2). With hydroxyacetals $\mathbf{2 j}$-l bearing neutral, moderately electron-rich, or electron-poor aryl substituents (entries 1-3), the corresponding benzo[b]carbazoles 4 were almost exlusively obtained. However, in the case of highly activated hydroxyacetals $\mathbf{2 m}, \mathbf{n}$, with a 2-thienyl or a trimethoxyphenyl group as $\mathrm{Ar}$ substituent, variable amounts of regioisomeric benzo $[b]$ carbazoles $\mathbf{4}^{\prime}$, differing on the final position of the methoxy group initially located at a defined position in the starting hydroxyacetal $\mathbf{2}$, were obtained (entries 4,5).

Table 2. Brønsted acid-catalyzed reaction of $N$ methylindole 1a with functionalized acetals $\mathbf{2 j}$-n. Competitive formation of benzo[b]carbazoles $\mathbf{4}$ vs $\mathbf{4}^{\prime}$ and $\mathbf{5}$.

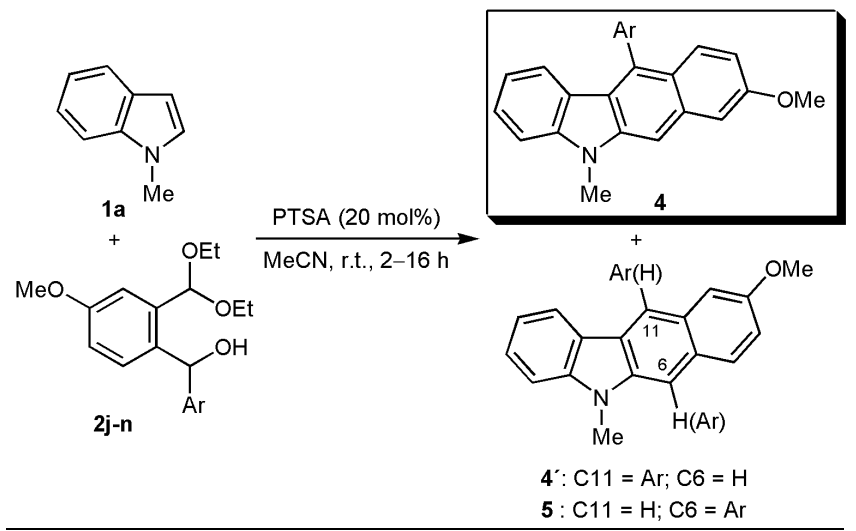

\begin{tabular}{lllll}
\hline Entry & $\mathbf{2}$ & $\mathrm{Ar}$ & Product(s) & $\begin{array}{l}\text { Yield } \\
{[\%]^{[\mathrm{b}]}}\end{array}$ \\
\hline 1 & $\mathbf{2 j}$ & $\mathrm{Ph}$ & $\mathbf{4 a j}$ & $60^{[\mathrm{c}]}$ \\
2 & $\mathbf{2 k}$ & $4-\mathrm{ClC}_{6} \mathrm{H}_{4}$ & $\mathbf{4 a k}$ & $68^{[\mathrm{c}]}$ \\
3 & $\mathbf{2 l}$ & $4-\mathrm{MeOC}_{6} \mathrm{H}_{4}$ & $\mathbf{4 a l}$ & $73^{[\mathrm{d}]}$ \\
4 & $\mathbf{2 m}$ & $2,3,4-(\mathrm{MeO})_{3} \mathrm{C}_{6} \mathrm{H}_{2}$ & $\mathbf{4 a m + 4} \mathbf{a m}(1 / 1.7)$ & $82^{[\mathrm{e}]}$ \\
5 & $\mathbf{2 n}$ & $2-\mathrm{Thienyl}$ & $\mathbf{4 a n + 4}$ an $(2 / 1)$ & $75^{[\mathrm{f}]}$ \\
\hline
\end{tabular}

[a] When two regioisomers were generated, the ratio (in brackets) was determined by ${ }^{1} \mathrm{H}$ NMR analysis of the crude reaction mixture. ${ }^{[b]}$ Yield of isolated product $\mathbf{4}$ based on the starting $N$-methylindole 1a. [c] ca. $5 \%$ of the corresponding 6-arylbenzo $[b]$ carbazole $\mathbf{5}$ was also isolated and characterized. ${ }^{[\mathrm{d}]}$ Trace amounts of $\mathbf{4}^{\prime} \mathbf{a l}$ and $5 \mathbf{a l}$ were also formed. ${ }^{[\mathrm{e}]}$ Yield for the mixture of regioisomers. The major one was isolated and characterized. ${ }^{[\mathrm{ff}} 56 \%$ of 4 an and $19 \%$ of 4 'an were isolated and characterized.

With all these results in hand, a catalytic cycle that could account for the formation of 
benzo $[b]$ carbazoles $\mathbf{4}^{\mathbf{4}^{\prime}}$, and $\mathbf{5}$ is shown in Scheme 3 . Initially, an equilibrium between hydroxyacetal 2 and cyclized acetal $\mathbf{6}$ is established in the acidic medium. Its reaction with the corresponding indole $\mathbf{1}$ would afford isobenzofuran derivatives $\mathbf{3}$, as it was observed when using several $\sigma$-Lewis acids or lower amounts of PTSA (see Table in Supporting Information). ${ }^{[21]}$ Probably favoured by the presence of the Brønsted acid these isobenzofurans $\mathbf{3}$ are in equilibrium with the corresponding 3,3'-BIMs 8 through the iminium intermediate 7 (path $a$ ). ${ }^{[22]}$ This hypothesis was supported by previous reports ${ }^{[12]}$ and by isolation of bisindole 8ab $\left(\mathrm{Ar}=4-\mathrm{ClC}_{6} \mathrm{H}_{4} ; \mathrm{G}=\mathrm{H}\right)$, along with 3ab, when carrying the reaction with $5 \mathrm{~mol} \%$ of PTSA for 30 minutes. Reactions under conditions reported in Table 1 (entry 2) of both isolated dihydroisobenzofuran $\mathbf{3 a b}$ and bisindole $\mathbf{8 a b}$ furnished benzo $[b]$ carbazole 4ab. Having proved the intermediacy of $\mathbf{3}$, as well as its equilibrium with $\mathbf{8}$ in the reaction media, the formation of benzo[b]carbazole 4 would be explained by an alternative path $b$ which would imply a nucleophilic addition of $\mathrm{C} 3$ of the indole that would generate spiro species 9. This key intermediate could undergo two different 1,2-alkyl shifts (Ciamician-Plancher rearrangement) to recover aromaticity after loss of a proton. ${ }^{[23]}$ Migration of the hydroxyalkyl group (path i) would lead to alcohol $\mathbf{1 0}$ that upon loss of water would afford benzo[b]carbazole 4 . On the other hand, a competitive migration of the benzylic group (path ii) would lead to regioisomeric alcohol 11 that would provide the corresponding benzo $[b]$ carbazole $\mathbf{5}$ after protonation and loss of water, probably through the iminium intermediate $\mathbf{1 2}$, and subsequent aromatization by further removal of a proton. However, both pathways do not account for the generation of benzo $[b]$ carbazoles $\mathbf{4}^{\prime}$, in which the relative position of the $\mathrm{G}$ substituent and the Ar group have changed with respect to the starting alcohol 2. So, we propose that the iminium intermediate $\mathbf{1 2}$ could also evolve through an alternative pathway involving a [1,4]-aryl migration. Subsequent loss of a proton would explain the formation of benzo $[b]$ carbazoles $4^{\prime}$. This competitive process seems to be partially operative exclusively when the hydroxymethyl group is located at C3 (intermediate 11 vs 10), probably due to the high stabilization of iminium intermediate 12. On the basis of this proposal the two 1,2-alkyl shifts (pathways $i$ and $i i$ ) could not be distinguised for non-functionalized hydroxyacetals $2(\mathrm{G}=\mathrm{H})$ as both of them colapse to the same product $\left(\mathbf{4}^{\prime}=\mathbf{4}\right.$ for $\left.\mathrm{G}=\mathrm{H}\right)$. From the results of Table 2 it seems that pathway $i$ is the preferred one whereas the benzyl migration (pathway ii) resulted to be competitive only for highly activated substrates $(\mathrm{G}$ $=\mathrm{OMe}$ and $\mathrm{Ar}=2-\mathrm{Th}$ or 2,3,4-(MeO) $\left.{ }_{3} \mathrm{C}_{6} \mathrm{H}_{2}\right)$ with an increased carbocation stabilization ability at the benzylic position. Also, the subsequent [1,4]-aryl migration on intermediate species $\mathbf{1 2}$ is favoured for electron-rich aromatics as Ar substituents. In other case, only trace amounts of regioisomers $\mathbf{5}$ are formed.

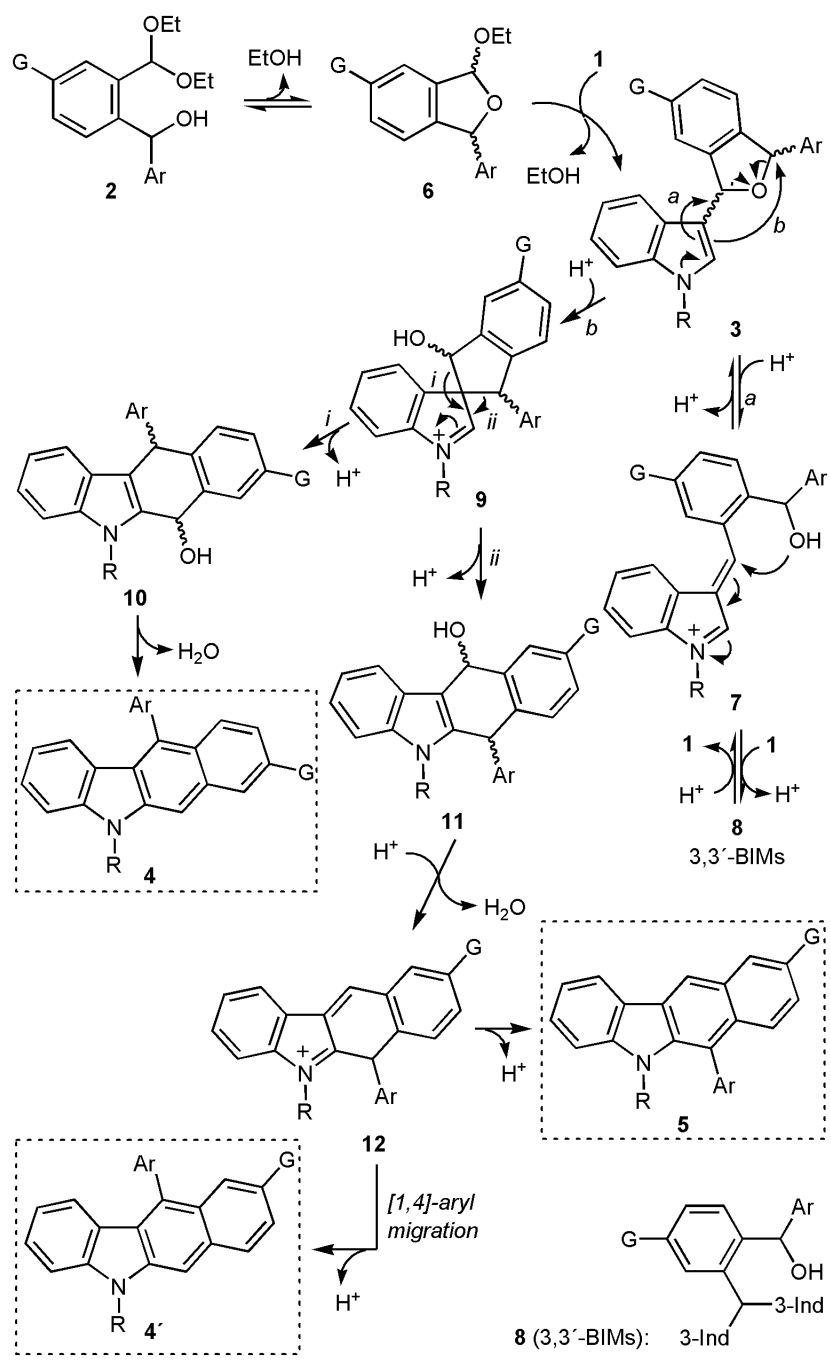

Scheme 3. Proposed mechanism for the formation of benzo $[b]$ carbazoles $\mathbf{4}, \mathbf{4}^{\prime}$, and $\mathbf{5}$.

Based in our proposed mechanism we envisioned that acetal derivatives $\mathbf{1 3}$ bearing a tertiary benzylic hydroxy group could be potential precursors of 6,11 disubstituted benzo[b]carbazoles thus expanding the scope of the reported benzocarbazole synthesis. Gratifyingly, treatment of indole 1a with hydroxyacetals 13a-c under the standard Brønsted acid catalysis selectively afforded the aimed 6,11disubstitued benzo $[b]$ carbazole derivatives 14 (that correspond with $\mathbf{4}^{\prime}$ in the general mechanism depicted in Scheme 3) in good yields (Scheme 4). ${ }^{[24]}$ It is interesting to note that for these acetals $\mathbf{1 3}$ the scope of the substitution at the benzylic carbon bearing the hydroxy group is not limited to aromatic substituents, like in secondary alcohols $\mathbf{2}$, as shown for 13ac having an alkynyl group that affords the corresponding benzo $[b]$ carbazole 14ac. As we anticipated, by increasing the migratory aptitude of the benzylic carbon in intermediate $\mathbf{1 5}$ (tertiary in $\mathbf{1 5}$ vs secondary in 9) an initial selective migration of the $t$-alkyl group (pathway ii in Scheme 3) occurred to form iminium species 16. These intermediates, in contrast with related ones $\mathbf{1 2}$ in Scheme 3, only could evolve by a formal [1,4]-migration to recover the 
aromaticity after loss of a proton (Scheme 4). Interestingly, the observed [1,4]-aryl or alkynyl shift has not precedent and opens the door to future developments in this field. Specially significant results the migration of an alkynyl group in the formation of 14ac as it is known the low migratory aptitude of these groups in carbocation rearrangements. ${ }^{[25]}$

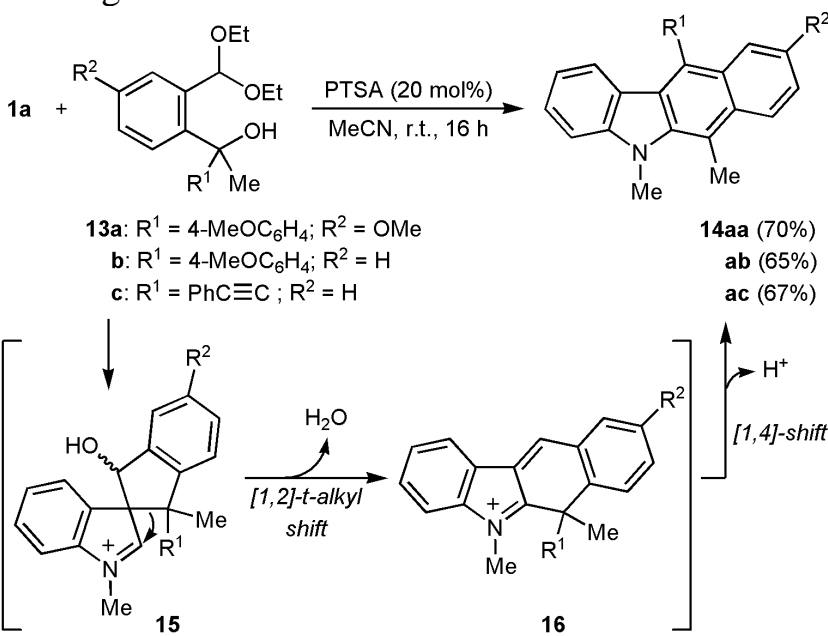

Scheme 4. Reactions of 1a with tertiary alcohols 13a-c. Synthesis of 6,11-disubstituted-5H-benzo[ $b]$ carbazoles $\mathbf{1 4}$.

Moreover, the reported methodology resulted to be also useful for the synthesis of heteroaryl-fused carbazoles. So, 10 -aryl-5H-thieno[3,2- $b$ ]carbazoles 18 were selectively prepared under the standard Brønsted acid-catalysis from thiophene-based hydroxyacetals 17a,b (Scheme 5). Interestingly, starting from regioisomeric hydroxyacetal 19, the corresponding thieno[2,3- $b]$ carbazole derivative $\mathbf{2 0}$, was exclusively formed (Scheme 5). In addition, benzo[4,5]thieno[2,3- $b$ ]carbazole $\mathbf{2 2}$ as well as benzofuro[2,3-b]carbazole $\mathbf{2 4}$ could also be prepared with this methodology from a properly functionalized benzo $[b]$ thiophene $\mathbf{2 1}$ and benzo[b]furan $\mathbf{2 3}$ respectively (Scheme 5). The formation of all of these carbazole derivatives could be understood in the same way as benzo[ $b]$ carbazoles $\mathbf{4}$, thus involving initial hydroxyalkyl shift (pathway $i$ in Scheme 3) followed by loss of water. Furthermore, the synthesis of these adducts was completely selective with the exception of carbazole derivative $\mathbf{2 4}$ which formation was accompanied with regioisomeric benzofuro[2,3$b$ carbazole 25 in a ca. 1.6:1 ratio (Scheme 5). ${ }^{[2]}$ On the contrary, hydroxyacetals bearing $\mathrm{N}$-heterocycles such as pyridines or indoles led to no reaction or decomposition under the established conditions (Figure 2). ${ }^{[27]}$
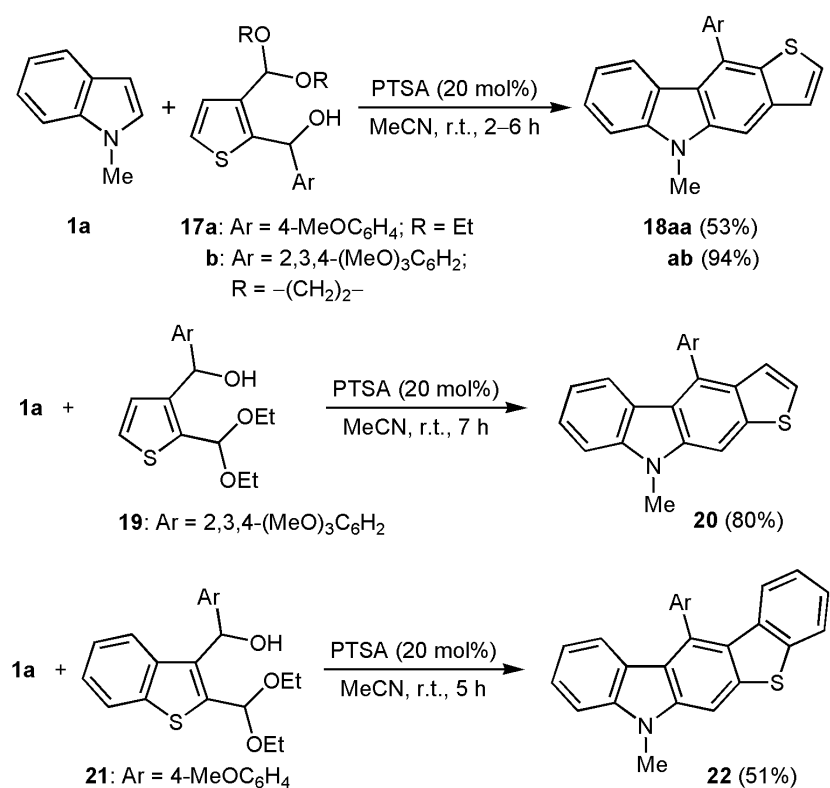

21: $\mathrm{Ar}=4-\mathrm{MeOC}_{6} \mathrm{H}_{4}$

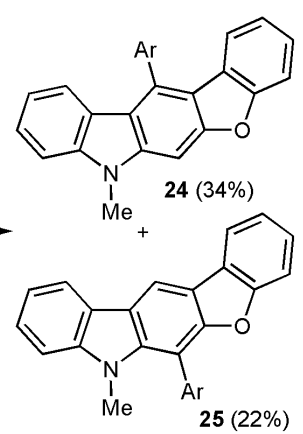

Scheme 5. Synthesis of heteroaryl $[b]$ carbazoles 18, 20, 22 , 24 and 25.
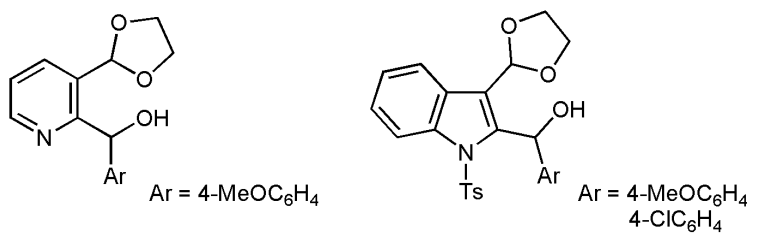

Figure 2. Not successful N-heterocycles-functionalized hydroxyacetals.

In conclusion, we have described a straightforward and regioselective synthesis of aryl-functionalized (hetero)aryl-annulated $[b]$ carbazoles from easily available starting materials such as indoles and $o-(\alpha-$ (hydroxy)benzyl)benzaldehyde acetals under simple Brønsted acid-catalysis through a new cascade sequence. This efficient and metal-free methodology affects the current synthetic scenario for the preparation of benzo $[b]$ carbazoles by adding a new strategy that allows the direct use of C2,C3unsubstituted indoles. Further studies to prove the proposed mechanism of this new transformation and to extend its synthetic scope are currently in progress in our laboratory. 


\section{Experimental Section}

\section{General Remarks}

All reactions involving air sensitive compounds were carried out under a $\mathrm{N}_{2}$ atmosphere $(99.99 \%)$. All glassware was oven-dried $\left(120{ }^{\circ} \mathrm{C}\right)$, evacuated and purged with nitrogen. All common reagents and solvents were obtained from commercial suppliers (VWR, Alfa and Aldrich) and used without any further purification. Solvents were dried by standard methods. Hexane and ethyl acetate were purchased as extra pure grade reagents and used as received. For the preparation of starting alcohols see the Supporting Information. TLC was performed on aluminum-backed plates coated with silica gel 60 with F254 indicator; the chromatograms were visualized under ultraviolet light and/or by staining with a Ce/Mo reagent and subsequent heating. $\mathrm{R}_{\mathrm{f}}$ values are reported on silica gel. Flash column chromatography was carried out on silica gel $60,230-240$ mesh. NMR spectra were measured on Varian Mercury-Plus $300 \mathrm{MHz}$ and Varian Inova-400 $\mathrm{MHz}$ spectrometers. High resolution mass spectra (HRMS) were recorded on a Micromass Autospec spectrometer using EI at $70 \mathrm{eV}$. Melting points were measured on a Gallenkamp apparatus using open capillary tubes and are uncorrected. GC-MS and low resolution mass spectra (LRMS) measurements were recorded on an Agilent 6890N/5973 Network GC System, equipped with a HP-5MS column.

\section{General procedure for the PTSA-catalyzed synthesis of benzo $[b]$ carbazoles $4,4^{\prime}$, and 5}

To a mixture of the corresponding acetal derivative 2 (1 $\mathrm{mmol})$ and the corresponding indole $\mathbf{1}$, in $\mathrm{MeCN}(1 \mathrm{~mL})$ was added PTSA (20 mol\%, $38 \mathrm{mg}$ ). The reaction mixture was stirred at room temperature until complete disappearance of the acetal derivative was observed by TLC $(0.5-24 \mathrm{~h})$, then it was quenched with a $0.5 \mathrm{M}$ aqueous solution of $\mathrm{NaOH}$, and extracted with EtOAc (3 $15 \mathrm{~mL})$. The combined organic layers were dried over anhydrous $\mathrm{Na}_{2} \mathrm{SO}_{4}$ and solvent was removed under reduced pressure. The remaining residue was purified by flash chromatography on silica gel using mixtures of hexane/EtOAc as eluents. The corresponding benzo $[b]$ carbazoles $\mathbf{4}, \mathbf{4}^{\prime}$, and $\mathbf{5}$ were isolated in the yields reported in the text. Characterization data and NMR spectra are presented in the Supporting Information.

\section{Acknowledgements}

We gratefully acknowledge the Ministerio de Economía $y$ Competitividad (MINECO) and FEDER (CTQ2010-15358) for financial suport. P.G.-G. and M.A.F.-R. thank MINECO for "Juan de la Cierva" and "Ramón y Cajal" contracts.

\section{References}

[1] For selected reviews, see: a) G. H. Kirsch, Curr. Org. Chem. 2001, 5, 507-518; b) H.-J. Knölker, D. R. Reddy, Chem. Rev. 2002, 102, 4303-4427; c) T. Janosik, N. Wahlström, J. Bergman, Tetrahedron 2008, 64, 9159-9180; d) A. W. Schmidt, K. R. Reddy, H.-J. Knölker, Chem. Rev. 2012, 12, 3193-3328.

[2] Isolation: a) S. Goodwin, A. F. Smith, E. C. Horning, J. Am. Chem. Soc. 1959, 81, 1903-1908; Anticancer activity: b) P.-L. Kuo, Y.-L. Hsu, C.-H. Chang, C.-C. Lin, Cancer Lett. 2005, 223, 293-301; Synthesis: c) G. W. Gribble, M. G. Saulnier, J. A. Obaza-Nutaitis, D. M. Ketcha, J. Org. Chem. 1992, 57, 5891-5899; d) J. M. Pedersen, W. R. Bowman, M. R. J. Elsegood, A. J.
Fletcher, P. J. Lovell, J. Org. Chem. 2005, 70, 10615-10618 and references cited therein.

[3] For selected reports, see: a) G. W. Gribble, M. G. Saulnier, J. Chem. Soc, Chem. Commun. 1984, 168-169; b) C. Asche, W. Frank, A. Albert, U. Kucklaender, Bioorg. Med. Chem. 2005, 13, 819-837; c) K. Kinoshita, T. Kobayashi, K. Asoh, N. Furuichi, T. Ito, H. Kawada, S. Hara, J. Ohwada, K. Hattori, T. Miyagi, W.-S. Hong, M.-J. Park, K. Takanashi, T. Tsukaguchi, H. Sakamoto, T. Tsukuda, N. Oikawa, J. Med. Chem. 2011, 54, 6286-6294.

[4] See, for example: a) N.-X. Hu, S. Xie, Z. Popovic, B. Ong, A.-M. Hor, J. Am. Chem. Soc. 1999, 121, 5097-5098; b) M. T. Levick, S. C. Coote, I. Grace, C. Lambert, M. L. Turner, D. J. Procter, Org. Lett. 2012, 14, 5744-5747; c) J.-Y. Balandier, N. Henry, J.-B. Arlin, L. Sanguinet, V. Lemaur, C. Niebel, B. Chattopadhyay, A. R. Kennedy, P. Leriche, P. Blanchard, J. Cornil, Y. H. Geerts, Org. Lett. 2013, 15, 302-305.

[5] a) G. W. Gribble, D. J. Keavy, D. A. Davis, M. G. Saulnier, B. Pelcman, T. C. Barden, M. P. Sibi, E. R. Olson, J. J. Belbruno, J. Org. Chem. 1992, 57, 5878-5891; b) H. L. Fraser, G. W. Gribble, Can. J. Chem. 2001, 79, 1515-1521; c) N. Haider, J. Käferböck, Tetrahedron 2004, 60, 6495-6507; d) P. Balczewski, A. Bodzioch, E. Rózycka-Sokolowska, B. Marciniak, P. Uznański, Chem. Eur. J. 2010, 16, 2392-2400; e) R. Sureshbabu, V. Saravanan, V. Dhalayan, A. K. Mohanakrishnan, Eur. J. Org. Chem. 2011, 922-935; f) V. Dhalayan, R. Sureshbabu, A. K. Mohanakrishnan, Indian J. Chem. 2011, 50B, 843-857; g) K. S. Prakash, R. Nagarajan, Adv. Synth. Catal. 2012, 354, 1566-1578.

[6] a) M. E. Budén, V. A. Vaillard, S. E. Martin, R. A. Rossi, J. Org. Chem. 2009, 74, 4490-4498; b) P. Appukkuttan, E. V. der Eycken, W. Dehaen, Synlett 2005, 127-133.

[7] a) M. Schmittel, J.-P. Steffen, M. A. W. Ángel, B. Engels, C. Lennartz, M. Hanrath, Angew. Chem. 1998, 110, 1633-1635; Angew. Chem. Int. Ed. 1998, 37, 1562-1564; b) C. Shi, K. K. Wang, J. Org. Chem. 1998, 63, 3517-3520; c) Xing, Y.; Hu, B.; Yao, Q.; Lu, P.; Wang, Y. Chem. Eur. J. 2013, 19, 12788-12793. See also ref. [2d].

[8] M. F. Martínez-Esperón, D. Rodríguez, L. Castedo, C. Saá, Tetrahedron 2008, 64, 3674-3686.

[9] Few particular examples have been reported: a) R.-Y. Tang, J.-H. Li, Chem. Eur. J. 2010, 16, 4733-4738; b) Y. Nagase, T. Miyamura, K. Inoue, T. Tsuchimoto, Chem. Lett. 2013, 42, 1170-1172.

[10] T. M. Kubczyk, S. M. Williams, J. R. Kean, T. E. Davies, S. H. Taylor, A. E. Graham, Green Chem. 2011, 13, 2320-2325.

[11] For a recent review, see: M. Shiri, M. A. Zolfigol, H. G. Kruger, Z. Tanbakouchian, Chem. Rev. 2010, 110, 2250-2293. Aryl(3-indolyl)carbenium ions I have been recently isolated as stable $o$-benzenedisulfonamide salts 
and fully characterized. See: M. Barbero, S. Cadamuro, F. Cauda, S. Dughera, G. Gervasio, P. Venturello, J. Org. Chem. 2012, 77, 4278-4287.

[12] a) F.-L. Sun, M. Zeng, Q. Gu, S.-L. You, Chem. Eur. J. 2009, 15, 8709-8712; b) H. Li, J. Yang, Y. Liu, Y. Li, J. Org. Chem. 2009, 74, 6797-6801.

[13] For a review, see: A. Palmieri, M. Petrini, R. R. Shaikh, Org. Biomol. Chem. 2010, 8, 1259-1270.

[14] a) R. Sanz, D. Miguel, J. Álvarez-Gutiérrez, F. Rodríguez, Synlett 2008, 975-978; b) R. Sanz, D. Miguel, A. Martínez, M. Gohain, P. García-García, M. A. Fernández-Rodríguez, E. Álvarez, F. Rodríguez Eur. J. Org. Chem. 2010, 7027-7039.

[15] Prepared from commercially available 2bromobenzaldehyde diethyl acetal by $\mathrm{Br}-\mathrm{Li}$ exchange and further reaction with benzaldehyde. See Supporting Information.

[16] For detailed optimization studies, see the Supporting Information.

[17] See, for example: A. C. Silvanus, S. J. Heffernan, D. J. Liptrot, G. Kociok-Köhn, B. I. Andrews, D. R. Carbery, Org. Lett. 2009, 11, 1175-1178.

[18] For pioneering work, see: C. Ferrer, A. M. Echavarren, Angew. Chem. 2006, 118, 1123-1127; Angew. Chem. Int. Ed. 2006, 45, 1105-1109. See, also: Y. Lu, X. Du, X. Jia, Y. Liu, Adv. Synth. Catal. 2009, $351,1517-1522$.

[19] For a recent report, see: C. C. J. Loh, G. Raabe, D. Enders, Chem. Eur. J. 2012, 18, 13250-13254, and references cited therein.

[20] The structure of all the new compounds $4, \mathbf{4}^{\prime}, \mathbf{5}, 14$ and $\mathbf{1 8}$ were determined by NMR techniques including COSY, NOESY and ${ }^{1} \mathrm{H}_{-}{ }^{13} \mathrm{C} 2 \mathrm{D}$ experiments. In addition, the substitution at $\mathrm{C} 6$ or $\mathrm{C} 11$ of the benzo $[b]$ carbazole moiety could be easily established as the substituent at $\mathrm{C} 11$ is significantly deshielded by the indole benzene ring. See ref. [5b] and [7b].

[21] The initial formation of isobenzofuran derivatives 3 precludes the possibility of an alternative mechanism involving a prior direct substitution of the hydroxy group by the indole through $\mathrm{C} 3$ and further direct cyclization by attack of $\mathrm{C} 2$ to the carbonyl.

[22] Indolation by Lewis acid-catalyzed $\mathrm{C}-\mathrm{O}$ bond cleavage: a) J. Barluenga, A. Fernández, F. Rodríguez, F. J. Fañanás, J. Organomet. Chem. 2009, 694, 546-550; b) X. Guo, S. Pan, J. Liu, Z. Li, J. Org. Chem. 2009, 74, 8848-8851.

[23] For selective 1,2-migration processes on spiroindolenine intermediates, see: a) G. Broggini, V. Barbera, E. M. Beccalli, E. Borsini, S. Galli, G. Lanza, G. Zecchi, Adv. Synth. Catal. 2012, 354, 159-170; b) A. S. K. Hashmi, W. Yang, F. Rominger, Adv. Synth. Catal. 2012, 354, 1273-1279; c) C.-X. Zhuo, Q.-F. Wu, Q. Zhao, Q.-L. Xu, S.-L. You, J. Am. Chem. Soc. 2013, 135, 8169-8172. For stereospecific migrations promoted by PTSA on spiroindolenines, see: d) Q.-F. Wu, C. Zheng, S.-L. You, Angew. Chem. 2012, 124, 1712-1715; Angew. Chem. Int. Ed. 2012, 51, 1680-1683; e) C. Zheng, Q.-F. Wu, S.-L. You, J. Org. Chem. 2013, 78, 4357-4365.

[24] The structure of 14aa was further confirmed by X-ray analysis. CCDC 959146 contains the supplementary crystallographic data for this compound. These data can be obtained free of charge from The Cambridge Crystallographic Data Centre via www.ccdc.cam.ac.uk/data_request/cif.

[25] G. E. Salnikov, A. M. Genaev, V. A. Bushmelev, V. G. Shubin, Org. Biomol. Chem. 2013, 11, 1498-1501.

[26] Whereas the formation of major regioisomer $\mathbf{2 4}$ would involve the expected pathway, i.e. hydroxyalkyl shift followed by loss of water, the minor product $\mathbf{2 5}$ seems to arise from a competitive hydroxyalkyl shift followed by $[1,4]$-aryl migration, probably due to the effect of the oxygen atom.

[27] Treatment of $\mathbf{1 a}$ with pyridine-functionalized hydroxyacetal (Figure 2) led to no reaction under catalytic $(20 \mathrm{~mol} \%)$ or excess $(120 \mathrm{~mol} \%)$ amounts of PTSA. 


\section{COMMUNICATION}

Brønsted Acid-Catalyzed Straightforward Synthesis of Benzo[b]carbazoles from 2,3-

Unsubstituted Indoles

Adv. Synth. Catal. 2013, 355, Page - Page

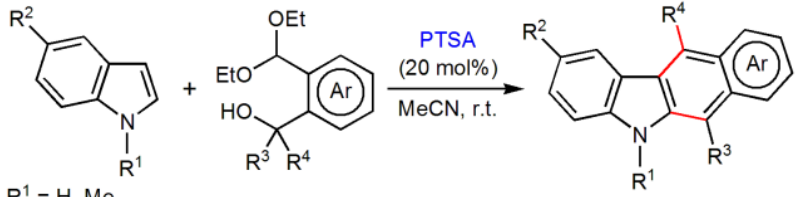

$\mathrm{R}^{1}=\mathrm{H}, \mathrm{Me}$

$\mathrm{R}^{2}=\mathrm{H}, \mathrm{Hal}, \mathrm{NO}_{2}, \mathrm{CO}_{2} \mathrm{Me}$

31 examples

$\mathrm{R}^{3}=\mathrm{H}, \mathrm{Me}$

up to $98 \%$ yield

Anisley Suárez, Patricia García-García, Manuel A.

$\mathrm{R}^{4}=\mathrm{Ar}$, alkynyl

Fernández-Rodríguez, Roberto Sanz* 